\title{
ÉT UDE COMPARATIVE DES PRINCIPALES CONSTANTES UTILISÉES POUR LA DÉTERMINATION DU MOUILLAGE DU LAIT
}

\author{
par J. CERF
}

Ingénieur de l'Institut agricole et diplômé de l'Ecole de Laiterie de la Faculté des Sciences de Nancy, Ancien assistant de l'Etablissement fédéral d'Industrie laitière et de Bactériologie du Liebefeld, Berne.

(Suite)

II ${ }^{e}$ PARTIE.

\section{B. LES CONSTANTES CHIMIQUES POUR LA DÉTERMINATION DU MOUILLAGE.}

M. Bouin [49], dans une "Etude des variations de la composition du lait de vache au cours de la lactation ", attire l'attention sur l'étendue des variations normales des composants du lait.

L'auteur a réuni un grand nombre de données analytiques, qui lui permettent de tirer les conclusions suivantes :

a) «Le taux de la matière grasse, variable dans le colostrum, est, en général, assez faible dans les laits de vaches fraîches à lait; puis il augmente progressivement du commencement à la fin de la période de lactation, tout en montrant un minimum très net aux mois de mai ou de juin, fait qui a été constaté antérieurement par Grorother, Hamoth, Hogxtröm, Ujhelgi, Eckles, Brioux, Richemont, etc. Les variations du taux de matière grasse peuvent être très étendues et dépasser même $50 \%$. )

b) «Le taux de matières protéiques totales, très élevé dans le colostrum, tombe rapidement et se maintient pendant les 5 ou 6 premiers mois, autour de $30 \mathrm{gr}$. par litre. Il augmente ensuite assez rapidement et peut atteindre et même dépasser $50 \mathrm{gr}$. dans le lait des vaches très vieilles à lait. Les variations du taux de matières protéiques peuvent donc atteindre normalement 30 à $40 \%$."

"La courbe de la caséine suit presque fidèlement celle des matières protéiques totales, et on peut dire que le taux de la caséine est sensiblement égal aux 8/10 du taux des matières protéiques totales."

c) “Les cendres brutes totales, très élevées dans le colostrum, s'abaissent rapidement jusqu'à un taux souvent inférieur à $7 \mathrm{gr}$. par litre. Ce taux se maintient sensiblement pencàant les 6 à 7 premiers mois de la lactation, avec quelques oscillations ; puis il augmente, lentement d'abord, puis rapidement en fin de lactation, où il atteint souvent 8 gr. 50 par litre, soit une variation de 18 à $20 \%$. Nous sommes loin de la constance remarquable que BORDAs et TOUPLAIN signalent. ,) 
d) " Le lactose, très faible dans le colostrum des premiers jours, augmente rapidement, pour atteindre un maximum généralement très voisin de $52 \mathrm{gr}$. par litre. La teneur en lactose diminue ensuite très lentement pendant les 6 à 7 premiers mois, puis plus rapidement, pour s'abaisser vers $42 \mathrm{ou} 43 \mathrm{gr}$. dans le lait de vaches très vieilles à lait. Nous avons donc ici des variations qui peuvent atteindre normalement 18 à $20 \%$, comme pour les cendres, mais en sens inverse. ")

L'auteur adopte la méthode de représentation graphique qu'il applique à différents composants au cours d'une période de lactation, et soumet également à la même épreuve plusieurs constantes chimiques. Nous avons repris et complété ce graphique (nous le donnons dans ce travail).

Les éléments constitutifs du lait sont si variables qu'ils ne sauraient servir de base à l'appréciation de sa pureté. Cependant, ces éléments ne varient pas tous dans le même sens au cours de la période de lactation. La matière grasse, les matières protéiques, la caséine et les cendres augmentent pendant tout le cours de la lactation (en dehors de la période colostrale), tandis qu'au contraire, le lactose diminue insensiblement. Mais la matière grasse oscille dans des limites trop variables pour qu'on puisse en tirer des indications précises. CoRNALBA estime ses variations à $48 \% ; \mathrm{M}$. BouIN, à plus de $50 \%$. Les matières protéiques totales, nous l'avons déjà dit, varient de 30 à $40 \%$. CORNALBA indique $41,4 \%$. Les variations des cendres et du lactose s'élèvent de 18 à $20 \%$. Ces deux éléments oscillent donc dans la même proportion, mais en sens inverse l'un de l'autre.

C'est à la lumière de ces faits, établis pour la première fois par M. BouIN, que nous allons étudier la valeur des différentes constantes chimiques.

\section{L'EXTRAIT SEC DÉGRAISSÉ OU CONSTANTE DE GROS.}

L'extrait dégraissé (E. D.) est la somme de lactose anhydre, des matières protéiques totales, des cendres réelles, de l'acide citrique et de l'indosé.

Il peut être déterminé avec suffisamment de précision par la formule de Fleischmann ou celle de Bouin et Gobert [58] :

$$
\text { E. D. }=0,2 \mathrm{~b}+2,665 \frac{100 \mathrm{~S}-100}{\mathrm{~S}}
$$

quand on opère sur $100 \mathrm{gr}$. de lait pour faire les dosages et

$$
\text { E. D. }=0,2 b_{1}+\frac{0,8}{3} \mathrm{D}
$$

quand on opère sur $100 \mathrm{~cm}^{3}$ de lait, formules dans lesquelles :

$\mathrm{b}=$ la matière grasse de $100 \mathrm{gr}$. de lait ; $\mathrm{S}=$ son poids spécifique à $15^{\circ} \mathrm{C}$. 
$b_{1}=$ la matière grasse de $100 \mathrm{~cm}^{3}$ de lait ; $\mathrm{D}=$ le degré densimétrique à $15^{\circ} \mathrm{C}$;

Comme l'ont démontré GoBERT et Bourn [50], quand l'opération doit avoir lieu sur des laits bichromatés, la densité est modifiée et, par suite, la formule donne des résultats moins précis, même en tenant compte de la correetion de la densité due à l'addition de cet antiseptique.

Il est nécessaire de déterminer chïmiquement l'extrait dégraissé. Au laboratoire, nous opérons selon la méthode de HINARD :

$10 \mathrm{~cm}^{3}$ de lait, additionnés de 5 gouttes d'un réactif contenant 1 partie de formol à $40 \%$ et 4 parties de $\mathrm{CH}^{3} \mathrm{COOH}$ à $25 \%$, sont desséchés à l'étuve à $65^{\circ} \mathrm{C}$., jusqu'à l'obtention de poids constant, c'est-à-dire 40 à 48 heures, dans une eapsule tarée de platine de $70 \mathrm{~m} / \mathrm{m}$ de diamètre. Après pesée, l'extrait sec total obtenu sert à la détermination des cendres. Pour obtenir l'E. D., on soustrait de l'extrait sec, la quantité de matière grasse pour $100 \mathrm{~cm}^{3}$ de lait, ainsi que la quantité de bichromate de $\mathrm{K}$.

Cette façon d'opérer sur des laits frais nous a toujours donné des résultats concordants et très voisins de ceux obtenus par la formule de Fleischmann.

L'E. D. est une "constante " dont les oscillations sont très fortes, en raison même des variations des éléments qui la composent. Sur le graphique, la courbe de l'E. D. a des écarts un peu plus faibles que les matières protéiques totales, parce que le lactose, variant en sens inverse, atténue ces oscillations.

Nous pouvons déterminer l'augmentation en poids de l'E. D. au cours de la lactation (période colostrale à part) par le calcul suivant :

Nous savons que l'E. D. est pratiquement la somme : Matières protéiques totales + Lactose + Cendres réelles.

Or les matières protéiques totales varient de 30 à 51 gr., soit

Les cendres réelles varient de 5,9 à 7,8

Le lactose varie de 52 à $43 \mathrm{gr}$

Augmentation

Diminution

...........

$21 \mathrm{gr}$.

$1,9 \mathrm{gr}$.

- $\frac{9 \mathrm{gr} .}{9 \mathrm{gr} .}$
$9 \mathrm{gr}$.

La différence des deux colonnes donne une augmentation de 13,9.

Sur le graphique, la courbe de l'E. D. a été transcrite d'après les différents dosages chimiques de cette constante; nous voyons que l'augmentation est sensiblement la même que celle que nous venons de calculer, c'est-à-dire $14 \mathrm{gr} .2$, soit une différence de $0 \mathrm{gr} .3$.

Ce calcul nous montre que l'augmentation de l'E. D. est due aux matières protéiques totales et aux cendres, insuffisamment corrigées par la diminution du lactose, d'où les oscillations de cette constante. Celle-ci, d'après M. Bourn [49], dépasse $20 \%$. 
Il est impossible de déterminer un mouillage de lait avec précision si l'on ne compare pas l'analyse avec un échantillon pris à l'étable. Nous ne sommes pas du tout d'accord avec L. GRos, quand il prétend que 95 fois sur $100, l^{\prime} \mathrm{E}$. D. varie de 90 à $102 \mathrm{gr}$. L'E. D. varie avec la race, la région, l'individu, l'âge du lait et même avec la nourriture de l'animal. En Lorraine, il atteint rarement $90 \mathrm{gr}$. par litre. Sa moyenne varie de 85 à $86 \mathrm{gr}$. Nous l'avons vu descendre très souvent audessous de ce chiffre. Autrefois on considérait comme movillé, tout lait ayant une constante Gros inférieure à 85 . Il est inutile d'insister sur le nombre considérable d'erreurs qui ont pu en être la conséquence. G. Cornalba [51] publie un certain nombre d'analyses pour démontrer la valeur de sa constante. Nous nous sommes permis, à titre de documentation, de calculer l'E. D. qui correspond à ces analyses.

A la page 35 : composition de laits (échantillons d'étables) provenant de troupeaux nombreux (de 15 jusqu'à plus de 100 têtes). Sur 30 échantillons, 14 analyses donnent un E. D. qui varie de 90,1 à 94,9 , et 16 autres un E. D. de 84,5 à 89,9 , avec une moyenne de 89,5 et un écart de $11 \%$.

A la page 36, composition de laits (échantillons d'étables) provenant de divers troupeaux. Sur 20 échantillons, 8 analyses donnent un E. D. qui varie de 90,17 à 91,65 , et les 12 autres de 86,13 à 89,74 , avec une moyenne de 89,127 et un écart de $6 \%$.

A la page 38 : composition de laits provenant d'individus isolés se trouvant dans des conditions diverses de lactation, d'alimentation, ete. Sur 26 échantillons, 9 analyses donnent un E. D. qui varie de 90 à $96,30,10 \mathrm{E}$. D. varient de 86,15 à 89,50 , et $7 \mathrm{E}$. D. varient de 81,13 à 84,62 . La moyenne est de 89,5 et l'écart de 15,5 .

L'E. D. varie beaucoup moins dans un même troupeau. Ainsi page 39, pour le troupeau No 1 , avee 4 analyses du 17 février 1908, nous n'avons plus qu'un écart de $1 \%$. Pour le No 2, l'expérience compte 10 analyses faites du 13 janvier 1908 au 14 avril 1908; l'écart est de $3,2 \%$. Pour le No 3 , pour 6 analyses du 13 janvier 1908 au 27 février 1908, l'écart est de $2,9 \%$.

A la page 40 : oscillations dans la composition des laits des individus :

\section{Dates}

Vache No 1.. 10.II.08 au 26.IV.08

Vache No 2, 10.II.08 au 26.IV.08

Vache No 3.. 10.II.08 au 26.IV.08

Vache No 4.. 10.II.08 au 26.IV.08
Nombres d'analyses

12

12

12

11
Chiffres extrêmes Ecarts

$\begin{array}{ll}82,09-88,08 & 6,8 \\ 83,6-88,50 & 5,5 \\ 86,2-90,80 & 5,0 \\ 86,00-94,30 & 8,75\end{array}$

Ces chiffres nous montrent qu'assez souvent 1'E. D. est inférieur à 85 et que, par conséquent, ce n'est pas la limite minimum. D'autre part, le chiffre 90 ne saurait être appliqué comme moyenne de l'E. D. devant servir, le cas échéant, au calcul du mouillage. La moyenne doit être déterminée dans chaque région et pour chaque saison. Nous sommes certain qu'il s'éloignera assez souvent de 90 .

Si nous prenons l'E. D. le plus bas et l'E. D. le plus élevé des ana- 
lyses de CoRnALBA [51], nous trouvons un écart de 15 à $16 \%$ sur des laits individuels.

En résumé :

Pour des laits de grands mélanges (laits de plusieurs centaines de vaches), l'E. D. peut servir avec une certaine sécurité à l'appréciation du mouillage, à la condition toutefois, qu'on ait déterminé soigneusement au préalable dans la région la moyenne de cette constante. Dans les cas des laits pathologiques, la constante Gros n'a plus de valeur.

Pour les analyses de laits individuels, I'E. D. ne peut, dans aucun cas, donner à lui seul des indications certaines sur la nature, la qualité et l'intégrité de l'échantillon. Même avec la comparaison du prélèvement à l'étable, l'E. D. seul ne suffit pas pour affirmer un mouillage, La courbe de cette constante sur le graphique le démontre. Les écarts d'un jour à l'autre, et pour un même groupe, peuvent dépasser $5 \%$.

Quoi qu'il en soit, l'E. D. doit être déterminé dans toutesles analyses de lait, où il apporte des renseignements précieux qui, confrontés avec d'autres, conduisent à la certitude dans l'interprétation des résultats.

\section{L'EXTRAIT DÉLACTOSÉ D'ACKERMANN.}

Cette constante ne'nous arrêtera pas longuement, car elle n'est et ne sera probablement jamais employée. On comprendra tout de suite pour quelle raison.

Comme son nom l'indique, c'est l'extrait dégraissé diminué du lactose. Rappelons que durant la lactation, le lactose corrige sensiblement les variations de l'E. D. AcKERMANN prend comme constante la somme des matières protéiques totales plus les cendres. Nous avons $v u$ que les matières protéiques présentaient des écarts de 30 à $40 \%$ et les cendres réelles de 24 à $25 \%$ dans le même sens. ACKERMANN supprime ainsi le terme correctif apporté par le lactose. Calculons pondéralement cette constante au cours de la lactation, comme nous l'avons fait pour l'E. D. :

Augmentation des matières protéiques ......... $21 \mathrm{gr}$.

Augmentation des cendres réelles ............ 1,9 gr.

$22,9 \mathrm{gr}$.

Un lait de vache "vieille à lait » ayant un extrait dégraissé de 99 gr. 2 et un lactose de $4.2 \mathrm{gr}$. par litre, pourrait être mouillé à $30 \%$ sans que cette constante révèle la fraude.

D'autre part, un lait de vache "fraîche à lait " ayant un E. D. de 85 gr, et un lactose de $50 \mathrm{gr}$. par litre, sera suspecté d'un mouillage de $12,5 \%$ par cette constante, sans qu'on ait fait aucune addition d'eau.

Passons la parole à M. Bourn [49] : "L'extrait délactosé proposé par ACKERMANN est tout à fait irrationnel. C'est pratiquement la 
somme : Protéiques totaux + Cendres. C'est en définitive prendre pour base de l'appréciation l'élément qui, normalement, présente dans le lait les variations les plus grandes, 30 à $40 \%$ (matière grasse exceptée, bien entendu).

"Prétendre que tous les laits purs doivent avoir un minimum de $40 \mathrm{gr}$. d'extrait délactosé, revient à dire que tous les laits doivent avoir un minimum de 32 à $33 \mathrm{gr}$. de matières protéiques, autrement dit, c'est vouer à la police correctionnelle presque tous les laits de vaches "fraîches à lait »." "

Nous le répétons: l'emploi de cette constante dans l'analyse du ait est à rejeter, car les résultats qu'elle donne ne pourraient qu'induire en erreur.

\section{LA CONSTANTE DE CORNALBA.}

Nous avons déjà cité, au sujet de l'E. D., le travail de G. CornalbA [51]. Cet auteur, après avoir observé que les matières solubles du lait ne variaient que de 5,9 à $6,6 \%$, en a déduit que e'était l'élément le plus constant, ainsi le plus propre à l'évaluation du mouillage. Le Cornalba est l'expression: E. S. T. (extrait sec total) - (M. G. + Caséine), autrement dit : extrait dégraissé moins la caséine.

Le système que nous avons employé précédemment, dans notre graphique, pour le calcul théorique de l'augmentation de 1'E. D. au cours de la lactation (période colostrale à part), peut s'appliquer à la constante de Cornalba.

L'E. D. augmente en cours de lactation de ...

A soustraire : l'augmentation de la caséine de
$13,9 \mathrm{gr}$.

$19 \mathrm{gr}$.

\section{Différence .}

$-5,1 \mathrm{gr}$.

Cette diminution agit directement sur la constante de Cornalba et révèle sa faiblesse dans les laits de vaches très "vieilles à lait \#. Elle représente un écart de $8,3 \%$.

En voici la preuve clairement formulée par M. BouIN : "Après la période colostrale, le Cornalba subira l'influence prépondérante du lactose et la courbe de cette constante sera sensiblement parallèle à celle du lactose. "

D'après CoRnALBA, le plus grand écart obtenu sur des vaches se trouvant dans des conditions diverses de lactation, d'alimentation, ete., est de 5,90 à 6,65 , soit $11,3 \%$. Mais il n'a pas étudié sa constante au cours de la lactation. Nous sommes certain que l'auteur reconnaitrait avec nous que l'écart de $11,3 \%$ n'est pas encore le chiffre maximum limitant les oscillations. Ainsi M. Bourn trouve un écart de 17,4 et même 17,9 en prenant les chiffres extrêmes du graphique.

CORNALBA [51] publie un certain nombre d'analyses où il recherche les laits les plus propres à l'allaitement naturel. Nous y trouvons 
des laits de vaches de différentes races et à des périodes de lactation différentes. Sa constante présente ici un écart de 5,573 à 6,496 , c'està-dire de 14,2\%.

A la page 468 , tableau $V$, on peut suivre sa constante durant la lactation de 3 vaches, du 10 février au 3 octobre :

La vache $N^{0} 1$ présente le 16 mai une constante de ...... 6,40 et

le 25 septembre $\ldots \ldots \ldots \ldots \ldots$. 5,90

soit un écart de $7,7 \%$.

La vache No 2, le 19 juillet, présente sa constante la plus forte ................... 6,36 et

le 13 août, présente sa constante lá plus faible .................... 5,77

soit un écart de $9,2 \%$.

La vache $\mathrm{N}^{\circ} 3$, le 19 avril $\ldots \ldots \ldots \ldots \ldots \ldots \ldots \ldots \ldots, 6,48$ et

le $16 \mathrm{mai} \ldots \ldots \ldots \ldots, \ldots \ldots \ldots \ldots, 5,58$

soit un écart de $13,6 \%$.

Telle est bien la démonstration pratique de ce que nous disions plus haut, e'est-à-dire que la constante de Cornalba présente un maximum. chez les vaches "fraîches à lait », et qu'elle diminue ensuite jusqu'à la fin de la lactation; c'est à ce moment qu'elle atteint son minimum.

Si nous comparons les variations de l'E. D. et celles de la constante de Cornalba dans les analyses que ce dernier publie dans la Revue générale du Lait, Nos 2 et 3,1908 , déjà cité, nous voyons :

Page 35 : Composition de laits (échantillons d'étables) provenant de troupeaux nombreux (minimum 15 têtes) :

Variation de C. C.

Variation de l'E. D. (Constante de

Cornalba)

30 analyses

$11 \%$

$7,8 \%$

Page 36 : Composition de laits (échantillons d'étables) provenant de divers troupeaux :

Variation de l'E. D. Variation de C. C.

20 analyses . . . . . . . . . . . $6 \%$.

Page 38 : Composition de laits provenant d'individus isolés se trouvant dans des conditions diverses de lactation et d'alimentation :

Variation de l'E. D. Variation de C.C.

26 analyses $\ldots \ldots \ldots \ldots \ldots \ldots \ldots . \quad 15,5 \% \quad 11,3 \%$

Page 39 : Oscillation dans la composition des laits d'un même troupeau :

Troupeau

No

Variation de l'E. D. Variation de C.C.

$\begin{array}{ll}1 \% & 3,1 \% \\ 3,2 \% & 4,5 \% \\ 2,9 \% & 5,5 \%\end{array}$


Page 40 : Oscillations dans la composition des laits d'individus isolés :

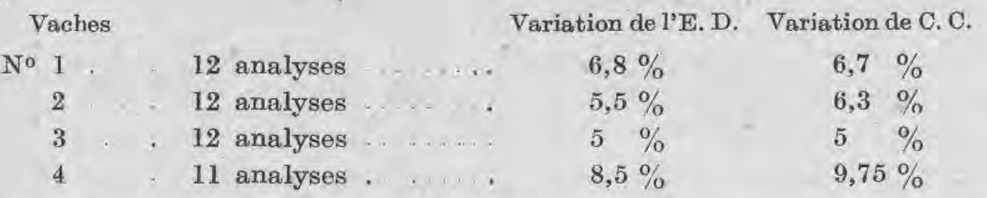

De ces chiffres, nous tirons les conclusions suivantes : Les écarts de ces deux constantes sont sensiblement les mêmes. En moyenne, 1'E. D. semble légèrement plus variable que les parties solubles.

La moyenne de la constante de Cornalba préconisée par l'auteur est 61,5 .

La moyenne préconisée par Wuyts et F. Courtay est 61,8 .

La moyenne préconisée par LAPIERRE est 65,4 .

Nous n'avons pas fixé nous-même la moyenne de cette constante pour les laits de Lorraine. Nous nous sommes contenté d'admettre le chiffre 6,15 indiqué par CORNALBA, chiffre que nous obtenons avec les laits de mélange. Nos expériences nous inciteraient à admettre une moyenne légèrement relevée.

Conclusions. - Ce que nous avons dit de l'E. D. est aussi vrai pour la constante de Cornalba. Néanmoins, les écarts de cette dernière sont moins grands, à cause de l'élimination de la caséine.

\section{CONSTANTE DE BORDAS ET TOUPLAIN.}

BORDas et TOUPLAIN [52], voulant appliquer la constante de Cornalba au procédé officiel d'analyses des laits, ont employé le réactif alcoolo-acétique pour précipiter les matières albuminoïdes. Ce réactif précipite avec la caséine une grande partie des matières protéiques. Le Cornalba devient donc :

Extrait sec dégraissé - Matières protéiques, que nous appellerons constante de Bordas et Touplain. Autrement dit, e'est la somme Lactose + Cendres. Cette constante est-elle supérieure ou inférieure à celle de Cornalba ? Le graphique nous montre qu'elle prêsente des écarts plus considérables que celle qui lui a donné naissance, et c'est inivitable. En effet, nous avons vu que dans la constante de Cornalba, l'écart provenant de la diminution du lactose était insuffisamment corrigé, d'une part, par l'augmentation des matières protéiques autres que la caséine et, d'autre part, par les cendres réelles. Dans la constante de Bordas et Touplain, il n'y a plus que l'augmentation des cendres réelles pour corriger le fléchissement du lactose.

A ce sujet M. Bourn [49] fait la remarque suivante :

"La faible proportion des cendres dans le lait n'aura que peu d'influence sur la constante de Bordas et Touplain, aussi, la courbe de cette constante est-elle presque rigoureusement parallèle à celle du 
lactose. A l'inverse de la précédente et à cause de l'élimination totale des protéiques, elle sera très faible dans les laits colostraux. »

Bordas et Touplain [52] indiquent des chiffres qui varient de 51,69 à 59,40 , soit un écart de $13 \%$ et une moyenne de 54,90. D'après notre graphique, l'écart est de $22,8 \%$ et les chiffres extrêmes vont de 47,50 à 60,00 .

Cette constante ne présentant aucun avantage particulier sur les constantes actuellement connues, nous ne voyons pas l'utilité de l'introduire dans une analyse de lait.

\section{LA CONSTANTE DE CONCENTRATION MOLÉCULAIRE SIMPLI- FIÉE DE MATHIEU ET FERRÉ (C.M.S.R.).}

Origine. - Ch. Porcher [54] écrivait en 1906: «De toutes les analyses de lait fournies par les nombreux auteurs qui ont voulu établir des moyennes, c'est le maximum de sucre de lait qui naturellement se trouve en face du minimum de sel et notamment du chlorure de sodium, c'est le minimum de lactose qui doit faire vis-à-vis au maximum de matières minérales, "

Frappés par la régularité avec laquelle les chlorures établissent l'équilibre osmotique du lait, Mathinu et FerRé [53] cherchent à évaluer en poids de lactose hydraté la somme du lactose et des chlorures du lait. Ces auteurs, s'appuyant sur les travaux de RAOULT, trouvent que $30 \mathrm{gr} .25$ de chlorure de sodium sont l'équivalent isotonique d'une molécule de lactose hydraté. En divisant le poids moléculaire du lactose 360 par 30,25 on obtient 11,9 , facteur de transformation du $\mathrm{NaCl}$ en lactose hydraté. C'est ainsi que ces auteurs créent la constante de concentration moléculaire simplifiée (C. M. S.) en faisant la somme du lactose hydraté et des chlorures, ces derniers étant évalués en $\mathrm{NaCl}$ et multipliés par 11,9.

Cette constante ne se base que sur deux éléments pour évaluer la concentration moléculaire du lait. Or nous savons que celle-ci ne dépend. pas uniquement du lactose et des chlorures, mais aussi d'une foule d'autres éléments, tels que citrates, phosphates et sulfates potassiques, bicarbonate de soude, azote non protéique et enfin l'ensemble salin des colloïdes. Done, la C. M. S. n'est que l'expression d'une partie de la concentration moléculaire du lait ou de son $\Delta$, e'est la raison pour laquelle Mathieu et FerRÉ l'ont appelée i simplifiée ".

Ch. Porcher [55] nous indique « la part qui revient à $\Delta$ de chaque composant du lait ». D'après lui, sont négligés dans la constante moléculaire simplifiée : 
3 gr. de eitrate ayant un $\Delta$ de........ - $0^{\circ} 027$ soit $5 \%$ du $\Delta$ total

2 gr. de phosphates mono- et bipotassique - $0^{0} 067$ " $12 \%$ "

0 gr. 15 de sulfate de $\mathrm{K}$ et de bicarbonate

de soude $\left(0\right.$ gr. 25) $\ldots \ldots \ldots \ldots \ldots \ldots-0^{\circ} 008$ » $1,5 \%$ "

1 gr. 5 d'azote non protéique $\ldots . \ldots \ldots+-0021$ " $4 \%$ n

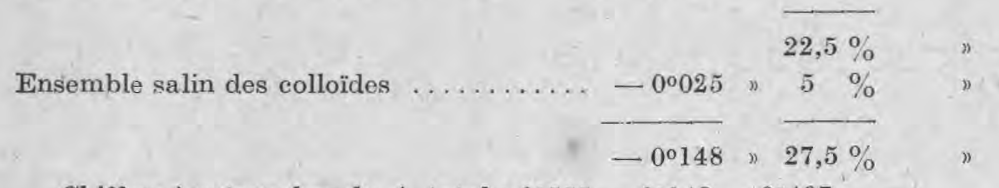

Chiffres à retrancher du $\Delta$ total $: 0^{\circ} 555-0^{\circ} 148=0^{\circ} 407$

La C. M. S. représente donc lès $\frac{407}{555}$ de $\Delta$, soit les $73,1 \%$; et en éliminant la part qui revient aux colloïdes, nous avons $\frac{404,5}{555}$, soit 72,8 .

Le même calcul peut se faire d'une façon plus simple en prenant un lait ayant un $\Delta$ moyen de $-0^{\circ} 555$ et une C. M. S. R. moyenne de 75 . D'après la formule $\frac{P}{M}=\frac{\Delta}{K}$, nous savons que 104 gr. 2 de lactose dissous dans la Q. S. d'eau pour faire $1.000 \mathrm{gr}$. ont un abaissement de température de $-0^{\circ} 555$. Ces 104 gr. 2 sont le chiffre que devrait avoir la C. M. S. R. (nous n'avons pas à tenir compte du volume de l'insoluble, la C. M. S. R. étant rapportée à 1.000 d'eau) puisqu'elle est l'expression pondérale du $\Delta$ en lactose hydraté. Dans le cas présent, elle atteint 75 ; elle ne représente done que $\frac{75}{104,2}$ du $\Delta$ total, soit $71,9 \%$.

Evidemment cette façon de calculer n'est qu'approximative, car pour des laits ayant un $\Delta$ de $-0^{\circ} 555$, nous pouvons tout aussi bien avoir une C. M. S. R. de 72 , de 76 ou de 78.

Nous avons passé trop rapidement sur l'influence du $\mathrm{NaCl}$ comme régulateur de l'équilibre osmotique entre le sérum du sang et celui du lait pour faire ressortir le rôle presque capital que joue cet élément vis-à-vis des fluctuations du lactose.

Aussi renvoyons-nous les lecteurs que la question intéresse au travail très complet de Ch. Porcher, paru dans la Revue générale du Lait, en 1906, et dans Le Lait, ${ }^{\circ} 1,1923$, p. 11, et auquel nous avons emprunté le principe qui figure en tête de ce chapitre. Si nous insistons sur ce sujet, e'est qu'il s'agit de la base physiologique de la C. M. S. R.

Détermination de la C. M. S. R. -
C. M.
R. $=[\mathrm{L}+(\mathrm{NaCl} \times 11,9)] \frac{1.000}{1.000-\mathrm{V}}$ 
dans laquelle C. M. S. R. = Constante moléculaire simplifiée réelle.

L

$\mathrm{NaCl}$

11,9

= Lactose hydraté pour $1.000 \mathrm{~cm}^{3}$ de lait.

$=$ Chlorure de sodium pour $1.000 \mathrm{~cm}^{3}$ de lait.

$=$ Facteur de transformation du $\mathrm{NaCl}$ en lactose hydraté.

1.000

$\overline{1.000-V}$ est une formule pour la correction de l'insoluble. dans laquelle :

$\mathrm{V}=\frac{\text { Matière grasse pour } 1.000 \mathrm{de} \text { lait }}{\text { Densité }(0,94)}+\frac{\text { Caséine pour } 1.000 \text { de lait }}{\text { Densité }(1,35)}$

Mathieu et FerRé ont établi un tableau donnant directement la valeur de $\frac{1.000}{1.000-V}$, que nous reproduisons ici.

\begin{tabular}{|c|c|c|c|c|c|c|c|c|c|c|c|}
\hline \multirow{2}{*}{$\begin{array}{l}\text { Caséine } \\
\mathrm{p}^{\mathrm{r}} \text { litre }\end{array}$} & \multicolumn{11}{|c|}{ Matière grasse pour 1.000 de lait } \\
\hline & 5 & 10 & 15 & 20 & 25 & 30 & 35 & 40 & 45 & 50 & 55 \\
\hline 40 & 1,036 & 1,041 & 1,047 & 1,053 & 1,059 & 1,065 & 1,071 & 1,077 & 1,084 & 1,090 & 1,096 \\
\hline 35 & 1,032 & 1,037 & 1,043 & 1,049 & 1,055 & 1,061 & 1,067 & 1,073 & 1,079 & 1,085 & 1,091 \\
\hline 30 & 1,029 & 1,033 & 1,039 & 1,045 & 1,051 & 1,057 & 1,063 & 1,069 & 1,075 & 1,080 & 1,087 \\
\hline 25 & 1,025 & 1,029 & 1,035 & 1,041 & 1,047 & 1,053 & 1,059 & 1,065 & 1,071 & 1,077 & 1,083 \\
\hline 20 & 1,020 & 1,026 & 1,031 & 1,037 & 1,043 & 1,048 & 1,054 & 1,060 & 1,066 & 1,073 & 1,079 \\
\hline
\end{tabular}

Au lieu d'utiliser le facteur $\frac{1.000-V}{1.000}$, nous pourrions aussi rapporter la C. M. S. à la quantité d'eau contenue dans le lait, au moyen de la densité et de l'extrait sec. La formule devient alors :

$$
\text { C. M. S. R. }=[\mathrm{L}+(\mathrm{NaCl} \times 11,9)] \frac{1.000}{1.000 \mathrm{D}-\mathrm{E} . \mathrm{S} . \mathrm{T}} \text {, où : }
$$

$\mathrm{D}=$ Densité du lait à $15^{\circ}(\mathrm{eau}=1)$.

E. S. T. = Extrait sec total en grammes par litre.

La première formule est plus exacte que la seconde, parce que l'extrait sec contient en poids toutes les matières solubles qui ne doivent pas entrer dans le calcul du volume du précipité. Cette correction du volume du précipité est nécessaire, parce que, si le $\Delta$ n'est pas influencé par les insolubles, il n'en est plus de même quand nous traduisons la concentration moléculaire en grammes de sucre. Quelle que soit la teneur en insoluble d'un lait, la concentration moléculaire reste la même, tandis que la quantité de cristalloïdes qui règle cette concentration est inversement proportionnelle au volume des matières en suspension. Quand celui-ci augmente, la quantité d'eau d'un litre de lait diminue et, avec elle, la quantité de sel dissous. L'inverse se 
produit aussi. C'est pour cette raison que l'écrémage n'influence pas le $\Delta$ d'un litre de lait, bien qu'il en augmente proportionnellement la quantité de sel dissous dans l'unité de volume. La C. M. S. R. étant l'expression pondérale du lactose et des chlorures du lait sera naturellement influencée par la plus ou moins forte proportion du volume du précipité. Pour éviter les oseillations auxquelles elle serait soumise, la formule reporte cette constante à la même quantité d'eau. C'est ce que nous appelons la constante de la concentration moléculaire simplifiée réelle (C. M. S. R.).

Valeur pratique de la C. IM. S. R. - Si cette constante était l'expression pondérale du $\Delta$ total du lait, il y a tout lieu de supposer que ses variations se limiteraient à celles de la cryoscopie et qu'ainsi elle en aurait la même valeur. Nous avons vu qu'elle ne représentait, selon Ch. Porcher, qu'environ les $3 / 4$ de $\Delta$. Il serait possible d'obtenir une eonstante moléculaire représentant le $\Delta$ intégral, en déterminant analytiquement tous les éléments qui donnent au point de congélation - 05555 , et de les transformer en lactose hydraté, en multipliant chacun de ces éléments par un coefficient spécial, comme il a été fait pour le $\mathrm{NaCl}$. Mais cette nouvelle constante de concentration deviendrait trop compliquée. Il serait alors infiniment plus simple de recourir directement à la détermination du point de congélation.

Sans vouloir prétendre qu'il est impossible de perfectionner encore la C. M. S. R., nous allons voir quelle est sa valeur réelle, en tant que " simplifiée ". Les écarts auxquels est soumise cette constante sont assez forts, comparativement anx chiffres obtenus avec d'autres constantes. Alors que nous avons évalué les écarts de la cryoscopie à $6 \%$, ceux de la C. M. S. R, atteignent 15 et même $20 \%$, suivant les auteurs.

Ch. Porcher signale des écarts de $12 \%$ (70 à 79$)$;

José GoLlan de $16 \%(70$ à 82$)$;

Monier-Williams de $20,5 \%$ (70 à 88,1$)$;

OBRÉ et MHe FournIER de $20 \%(65,9$ à 81,7$)$.

Il est évident que d'aussi fortes variations peuvent laisser passer des mouillages aussi considérables que ces écarts; dans certains laits à C. M. S. R. fortes. D'autre part, cette constante perd aussi toute séeurité dans les cas de laits pathologiques. Aussi ne partageons-nous pas la haute opinion qu'a Ch. Poroher de l'excellence de la C. M. S. R. En effet, cet auteur dit dans Le Lait (juin 1923, p. 452) : "Que la mamelle soit atteinte et sécrète par conséquent un lait vraiment pathologique, ou que la mamelle ne le soit pas, bien qu'appartenant à un individu malade, la constante moléculaire simplifiée n'est pas modifiée. "Nous ne sommes pas d'accord, et pour cause, avec cette affirmation. Nous nous en référons simplement aux résultats de nos expériences. Nous avons calculé la C.M. S. R. sur quelques analyses 
fournies par des auteurs ayant étudié la composition des laits pathologiques.

D'après Hess, Schaffer et Bondzynski (Landw. Jahrbuch der Schweiz, 1888):

Lait provenant de vaches atteintes de:

\begin{tabular}{|c|c|c|c|c|c|}
\hline & $\begin{array}{c}\text { Lactose } \\
0_{0}, 0\end{array}$ & $\underset{\%}{\mathrm{NaCl}}$ & $\begin{array}{c}\text { Albumine } \\
\%\end{array}$ & $\begin{array}{c}\text { M. G. } \\
0_{i 0}\end{array}$ & C. M. S. R. \\
\hline Mammite non infectieuse & 0,53 & 0,422 & 4,01 & 0,82 & 47,68 \\
\hline Mammite parenchymateuse & 1,01 & 0,444 & 4,21 & 2,16 & 66,48 \\
\hline \multicolumn{6}{|l|}{$\begin{array}{l}\text { D'après STORCH : } \\
\text { Vache III }\end{array}$} \\
\hline Quartier malade (2.VII.) & 0,00 & 0,74 & 5,22 & 1,20 & 88,06 \\
\hline Quartier sain ......... & 0,40 & 0,50 & 11,59 & 11,79 & 78,10 \\
\hline \multicolumn{6}{|c|}{ D'après Monvoisin (Le lait et ses produits dérivés, p. 178 et 179) : } \\
\hline Vache IV & $\begin{array}{l}\text { Lactose } \\
\quad \%\end{array}$ & $\begin{array}{l}\mathrm{NaCl} \\
\%\end{array}$ & $\begin{array}{l}\text { Albumine } \\
\%\end{array}$ & $\begin{array}{l}\text { M. G. } \\
\%\end{array}$ & C. M. S. R. \\
\hline Quartier malade (1.X.). & $2 ; 50$ & 0,33 & 3,03 & 3,88 & 68,44 \\
\hline Quartier sain & 3,48 & 0,15 & 2,68 & 3,15 & 55,54 \\
\hline Quartier malade (21.X.). & 1,27 & 0,42 & 3,42 & 5,33 & 65,81 \\
\hline Quartier sain ......... & 3,16 & 0,19 & 2,73 & 10,80 & 62,17 \\
\hline
\end{tabular}

A ces exemples, nous pourrions ajouter de nombreux autres cas semblables que nous avons calculés sur des analyses publiées dans différents ouvrages. Nous nous contenterons, pour ne pas allonger démesurément ce chapitre, de citer encore nos propres résultats :

VACHE “ RIGA " ATTEINTE DE MAMMITE STREPTOGOCGIQUE (1).

Analyse du 20.II.30 Analyse du 26.II.30 Analyse du 13.II.30

Quartier devant

gauche

Lactose hydraté .....

Chlorure de sodium . .

Caséine ...........

Matière grasse .....

C. M. S. R. .......

\section{$0,255 \mathrm{gr} . \% \mathrm{~cm}^{3}$}

3,463 gr. $\% \mathrm{~cm}^{3}$ $4,862 \mathrm{gr} . \% \mathrm{~cm}^{3}$

1,22 gr. $\% \mathrm{~cm}^{3}$

46,10
Quartierdevant gauche
Quartier devant droit

\section{VACHE " TRÉSA " ATTEINTE D'UN REFROIDISSEMENT.}

Quartier derrière gauche

Début de la traite
Fin de la traite

Lactose ? hydraté $\{4,8$

Chlorure de sodium $\}$

\begin{tabular}{|c|c|c|}
\hline Caséine & $3,419 \mathrm{gr} . \% \mathrm{~cm}^{3}$ & $3,406 \mathrm{gr} . \% \mathrm{~cm}^{3}$ \\
\hline $\begin{array}{c}\text { Matière } \\
\text { grasse }\end{array}$ & $1,75 \mathrm{gr} . \% \mathrm{~cm}^{3}$ & $4,08 \mathrm{gr} . \% \mathrm{~cm}^{3}$ \\
\hline 1 & 68,20 & 71,0 \\
\hline
\end{tabular}

C.M.S.R. 68,20

Quartier devant droit

Début de la traite

Fin de la traite

(1) Voir le détail dés analyses à la fin du travail. 
Nous avons vu que même le point de congélation peut être en défaut. Comment alors la C. M. S. R. ne le serait-elle pas ? Du reste, les chlorures sont-ils seuls à augmenter dans les cas pathologiques résultant plus spécialement des troubles de la mamelle ? Il y a longtemps que Schnorf [37], Bergema, Koestler [56] nous ont démontré que les substances. azotées, non protéiques, augmentaient également. Nous savons aussi, par les mêmes auteurs, qu'avec le lactose diminuent la caséine, les citrates, les phosphates et la potasse. La diminution de ces éléments est-elle compensée dans le $\Delta$ par l'augmentation de l'albumine, de la globuline et de l'azote non protéique?

Cédons encore une fois la parole à Ch. PorCher et empruntons-lui son tableau sur "Les composants du lait selon leur signification et leur origine $n$ :

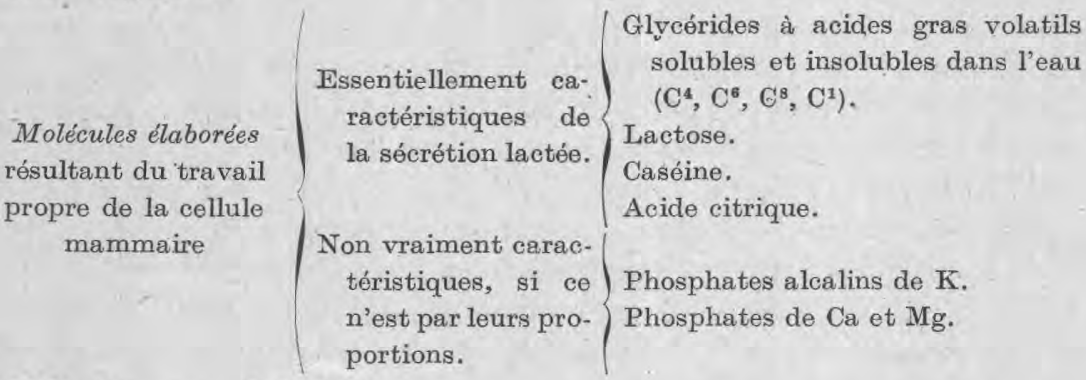

Molécules non élaborées ne sont pas particulières au travail de la cellule mammaire.

Albumine et globuline.

Azote non protéique.

Chlorure de sodium.

Chaque fois que le fonctionnement mammaire est entravé, la quantité des molécules non élaborées augmente, tandis que celle des molécules élaborées diminue.

Ce phénomène est mis en évidence dans nos analyses des vaches "Parma » et "Riga ", analyses détaillées à la fin du présent travail.

\title{
CONSIDÉRATIONS PHYSICO-CHIMIQUES SUR LE FROMAGE
}

\author{
par G. KOEȘTLER
}

Travail de l'Etablissement fédéral d'Industrie Laitière et de Bactériologie, Liebefeld-Berne. (Fin:)

VI. MÉTHODOLOGIE.

L'analyse physique de la masse de fromage mûre en est encore à débuts. En dehors des recherches faites occasionnellement sur 